\title{
Constraining the Accretion Geometry of the Intermediate Polar EX Hya Using NuSTAR, Swift, and Chandra Observations
}

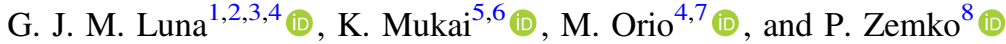 \\ ${ }^{1}$ CONICET-Universidad de Buenos Aires, Instituto de Astronomía y Física del Espacio, (IAFE), \\ Av. Inte. Güiraldes 2620, C1428ZAA, Buenos Aires, Argentina gjmluna@iafe.uba.ar \\ ${ }^{2}$ Universidad de Buenos Aires, Facultad de Ciencias Exactas y Naturales, Buenos Aires, Argentina \\ ${ }^{3}$ Universidad Nacional Arturo Jauretche, Av. Calchaquí 6200, F. Varela, Buenos Aires, Argentina \\ ${ }^{4}$ INAF-Osservatorio di Padova, vicolo dell Osservatorio 5, I-35122 Padova, Italy \\ ${ }^{5}$ CRESST and X-ray Astrophysics Laboratory, NASA Goddard Space Flight Center, Greenbelt, MD 20771, USA \\ ${ }^{6}$ Department of Physics, University of Maryland, Baltimore County, 1000 Hilltop Circle, Baltimore, MD 21250, USA \\ 7 Department of Astronomy, University of Wisconsin, 475 N. Charter Street, Madison, WI 53704, USA \\ ${ }^{8}$ Department of Physics and Astronomy, Universit'a di Padova, vicolo dell Osservatorio 3, I-35122 Padova, Italy \\ Received 2017 November 10; revised 2017 December 7; accepted 2017 December 14; published 2018 January 2
}

\begin{abstract}
In magnetically accreting white dwarfs, the height above the white dwarf surface where the standing shock is formed is intimately related with the accretion rate and the white dwarf mass. However, it is difficult to measure. We obtained new data with NuSTAR and Swift that, together with archival Chandra data, allow us to constrain the height of the shock in the intermediate polar EX Hya. We conclude that the shock has to form at least at a distance of about one white dwarf radius from the surface in order to explain the weak Fe $\mathrm{K} \alpha 6.4 \mathrm{keV}$ line, the absence of a reflection hump in the high-energy continuum, and the energy dependence of the white dwarf spin pulsed fraction. Additionally, the NUSTAR data allowed us to measure the true, uncontaminated hard X-ray $(12-40 \mathrm{keV})$ flux, whose measurement was contaminated by the nearby galaxy cluster Abell 3528 in non-imaging X-ray instruments.
\end{abstract}

Key words: novae, cataclysmic variables - radiation mechanisms: general - X-rays: individual (EX Hydrae)

\section{Introduction}

In magnetic cataclysmic variables (CVs) the primary is a highly magnetized $\left(B \gtrsim 10^{6} \mathrm{G}\right)$ white dwarf (WD) whose field controls the accretion flow close to the $\mathrm{WD}$, leading to shock and accretion columns that radiate chiefly in X-rays. The shock temperature $k T_{\text {sh }}$ is determined by the pre-shock velocity and is of the order of $10-50 \mathrm{keV}$ in magnetic CVs. The post-shock plasma must further decelerate and cool before it can settle onto the WD. Thus, the height of the shock $\left(h_{\mathrm{sh}}\right)$ is determined by equating the plasma cooling time with the remaining travel time from the shock front to the WD surface (Aizu 1973). Since the X-ray cooling time is inversely proportional to the density of the post-shock plasma, $h_{\mathrm{sh}}$ is small if the accretion rate per unit area (or specific accretion rate) is high. If $h_{\mathrm{sh}}$ is a small fraction of the white dwarf radius $\left(R_{\mathrm{WD}}\right)$, and if the accretion flow can be considered to be freefalling from infinity, then $k T_{\text {sh }}$ is an immediate indicator of the white dwarf mass $\left(M_{\mathrm{WD}}\right)$. This works well for most intermediate polars (IPs). However, if the specific accretion rate is low, $h_{\text {sh }}$ may not be negligible. This would reduce the freefall velocity above the shock, and hence $k T_{\text {sh. }}$. Also, if accretion is from a truncated disk with a small inner radius $R_{\text {in }}$, the pre-shock velocity is set by the freefall condition from $R_{\mathrm{in}}$, requiring a different correction (Suleimanov et al. 2005; Luna et al. 2015).

The subject of this Letter, EX Hya, is a unique IP that has raised several important, and still unresolved questions. One is the very nature of its accretion flow. A standard, Keplerian, partial disk cannot be present in this system if the WD is in spin equilibrium, because $R_{\text {in }}$ (given the long spin period, 67 minutes, relative to the orbital period, 98 minutes) would be so large as to violate the physical condition for the formation of a disk (King \& Lasota 1991). Either the WD is far out of equilibrium, or EX Hya possesses a diamagnetic blob/ring type structure between the magnetosphere and L1 (King \& Wynn 1999; Norton et al. 2008).

The other major unresolved question is why the X-ray spectrum of EX Hya is so soft. The combination of partial eclipse and optical spectroscopy has led to an estimate of $M_{\mathrm{WD}}=0.78 \pm 0.03 M_{\odot}$ (Echevarria et al. 2016), implying $k T_{\text {sh }} \sim 35 \mathrm{keV}$ in the Aizu picture, while X-ray measurements are consistently below $\sim 20 \mathrm{keV}$ (see, e.g., Luna et al. 2015). This can be resolved by either having a small $R_{\mathrm{in}}$ or a large $h_{\mathrm{sh}}$.

In EX Hya, $h_{\mathrm{sh}}$ has been inferred through indirect arguments and some of them have yielded opposing answers. Allan et al. (1998) studied the spin modulation and the partial eclipse in the ASCA data, and argued for a tall $\left(h_{\mathrm{sh}} \sim 1 R_{\mathrm{WD}}\right)$ shock as the explanation for the spin modulation, and also a large $\left(R_{\text {in }}>\right.$ $\left.6.1 R_{\mathrm{WD}}\right)$ inner disk radius. A smaller $R_{\mathrm{in}}$ would result in the accretion disk blocking our view of the lower pole. Other arguments supporting that $\mathrm{R}_{\mathrm{in}}>$ a few $R_{\mathrm{WD}}$ and that thus $h_{\mathrm{sh}}$ is a non-negligible fraction of $R_{\mathrm{WD}}$ are: (i) the equilibrium spin period is expected to be close to the Keplerian period at $R_{\mathrm{in}}$; for small values of $R_{\text {in }}$, the spin period would be smaller than the observed $67 \mathrm{~m}$; and (ii) Hellier et al. (1987) analyzed an extensive set of optical spectra and found three components in the line profile: a narrow $S$-wave component; a double-peaked component; and a broad, spin-modulated component. Measuring the width at spin phase 0.5 allowed them to put an upper limit on the high-velocity extent of the double-peaked, presumably accreting disk, concluding that for a $0.78 M_{\mathrm{WD}}$, $R_{\text {in }}$ is about $10 R_{\mathrm{WD}}$.

On the other hand, Revnivtsev et al. (2011) and Semena et al. (2014) modeled the break frequency in the power spectrum of stochastic variability and proposed $R_{\mathrm{in}}=2.7 R_{\mathrm{WD}}$. Belle et al. (2003), Suleimanov et al. (2016) and Echevarria et al. (2016) also derived small $R_{\text {in }}$ using other methods. This small $R_{\text {in }}$ would reduce $k T_{\text {sh }}$ : this was the solution preferred by 
Luna et al. (2015), who analyzed high-quality, half a megasecond, Chandra HETG data on EX Hya and found a number of discrepancies with respect to the standard models of X-ray emission. A tall shock would imply that gravity adds heat to the cooling flow region and the magnetic field geometry adds heat by magnetic pressure. Both mechanisms should modify the emission measure distribution of the cooling plasma, increasing both the flux of $\mathrm{H}$ - and He-like lines. Such models did not match the Chandra/HETG observations.

An observational determination of $h_{\mathrm{sh}}$ is essential for deciding which picture is correct. Because of the accretion geometry, we expect the X-rays emitted in the post-shock region to be reflected back into our line of sight by the WD surface, producing a detectable Compton hump at energies above $10 \mathrm{keV}$ (e.g., Hayashi et al. 2018). The detection of the reflection feature in three IPs has been possible only recently with NUSTAR (Mukai et al. 2015). Constraining the presence of a reflection component in the hard X-ray spectrum of EX Hya would allow us to determine $h_{\mathrm{sh}}$. A tall shock implies little-tono reflection with a weak $\mathrm{Fe} \mathrm{K} \alpha 6.4 \mathrm{keV}$ fluorescence line and small or non-existent spin modulation above a few $\mathrm{keV}$, since photoelectric absorption on the order of $10^{22} \mathrm{~cm}^{-2}$ cannot affect the light curves at these energies (the measured $N_{H}$ is even lower) and the shock region would not be hidden by the WD body. On the other hand, a negligible shock height would imply a strong reflection amplitude and a strong $\mathrm{Fe} \mathrm{K} \alpha$ line, while the spin modulation would be almost entirely due to absorption and thus the pulsed fraction of the light curves should be a strong function of the energy. Somewhere in the middle, a shock height of a non-negligible fraction of the WD radius $\left(\sim 0.1-0.5 R_{\mathrm{WD}}\right)$ would imply a moderate reflection amplitude (less than 1), potentially detectable with NuSTAR. The modulation of the low-energy X-rays $(E \sim 5-10 \mathrm{keV})$ would be mostly due to occultation of the accretion column by the body of the WD, while the expected modulation at higher energies would be entirely due to occultation, so the pulsed fraction at high energies should not be a function of energy. To perform this test, we have observed EX Hya with $N u S T A R$.

There is an additional reason why it is important to perform a NuSTAR observation of EX Hya. While it was proposed to be the counterpart of a Uhuru source from the early days of X-ray astronomy (Warner 1972), and it is indeed the brightest source in the traditional $(0.5-10 \mathrm{keV}) \mathrm{X}$-ray band among all CVs, it is not the only bright X-ray source in this region of the sky. The interacting cluster of galaxies, Abell 3528, consists of two X-ray bright subclusters (Gastaldello et al. 2003), located $\sim 29$ arcmin from EX Hya. Therefore, the possibility of contamination must be kept in mind for interpreting any nonimaging X-ray observations of EX Hya. This means, in part, that there have been no reliable observations of EX Hya above $10 \mathrm{keV}$ until now.

In this Letter, we present new contemporaneous $N u S T A R$ and Swift observations of EX Hya, and new analysis of archival Chandra observation. We present conclusive arguments for an absence of reflection in the X-ray spectrum, implying that the height of the shock must be an appreciable fraction of the size of the WD. In Section 2 we detail the reduction of NuSTAR, Swift, and Chandra data, while Section 3 presents the results from the spectral and timing analysis. Finally, Section 4 presents a discussion about the implications of the nondetection of reflection for the structure of the accretion column.

\section{Observations}

We observed EX Hya with NuSTAR on 2016-06-05 for $24.8 \mathrm{ks}$. The data were reduced using the NuSTAR Data Analysis Software as part of HEASOFT 6.21 and filtered using standard filters given that the observation was not affected by abnormal solar activity. Using the tool nuproducts we extracted source spectra, baricenter-corrected source event files, and light curves from a circular region centered on the SIMBAD coordinates, $\alpha=12^{\mathrm{h}} 52^{\mathrm{m}} 18.5, \delta=-29^{\circ} 16^{\prime} 16^{\prime \prime}$ in the FPMA chip and $\alpha=12^{\mathrm{h}} 52^{\mathrm{m}} 23^{\mathrm{s}} .8, \delta=-29^{\circ} 14^{\prime} 55^{\prime \prime}$. $^{\prime}$ in the FPMB chip, with a $30^{\prime \prime}$ radius. For the background, we choose an annular region with inner and outer radii of $110^{\prime \prime}$ and $220^{\prime \prime}$, respectively, and centered on the respective source coordinates.

A Swift observation was obtained almost simultaneously with $N u S T A R$, with $1.8 \mathrm{ks}$ exposure time. We extracted source $\mathrm{X}$-ray spectra from a circular region with a radius of 20 pixels centered on the SIMBAD coordinates. We extracted background events from an annular region with inner and outer radii of 25 and 40 pixels, respectively. We built the ancillary matrix (ARF) using the tool xrtmkarf and used the swxpc0to12s6_20130101v014.rmf response matrix provided by the Swift calibration team.

EX Hya was observed with Chandra using the ACIS-S/ HETG combination for $496 \mathrm{ks}$ and the spectral analysis has been already described in Luna et al. (2010; see also Luna et al. 2015). For the present study, the events arrival times were barycentrically corrected using the axbary script and filtered to extract event arrival times from the source in the energy regions of interest (strongest emission lines), selecting only HEG and MEG \pm 1 orders.

\section{Results}

\subsection{Spectral Model}

We first modeled the continuum of the NUSTAR spectrum excluding the $\mathrm{Fe}$ and $\mathrm{Ni}$ lines region (5-9 $\mathrm{keV}$ ) and because both internal and interstellar absorption are known to be small in EX Hya $\left(\lesssim 10^{21} \mathrm{~cm}^{-2}\right)$, we only used a single-temperature model modified by reflection. First, we used solar abundances for the reflecting plasma. ${ }^{9}$ This model is statistically acceptable, with $\chi_{\nu}^{2}=1.06 / 187$ dof, a temperature of $k T=9.9 \pm 0.6 \mathrm{keV}$, and an unconstrained reflection amplitude of $<0.29$ (see Table 1). However, most X-ray data indicate sub-solar Fe abundances (e.g., Allan et al. 1998; Luna et al. 2015), with $\approx 60 \%$ the solar value. Fixing the Fe abundance to 0.60 yielded a similarly acceptable fit with $\chi_{\nu}^{2}=1.06 / 187$ dof, $k T=$ $9.9 \pm 0.6$ and an equally unconstrained reflection amplitude.

We also tested models where we included back the Fe and $\mathrm{Ni}$ lines regions and used a variable abundance, multi-temperature plasma (vmcflow), plus a Gaussian line to account for the presence of the $\mathrm{Fe} \mathrm{K} \alpha 6.4 \mathrm{keV}$ fluorescence line. First, fixing the $\mathrm{Fe}$ abundance to 0.60 ( $\mathrm{Ni}$ abundance are tied to $\mathrm{Fe}$ in our fits) yielded a maximum temperature of $k T_{\max }=13.4 \pm$ $0.8 \mathrm{keV}$ and a reflection amplitude of $1.24 \pm 0.33$, with $\chi_{\nu}^{2}=1.19 / 347$ dof. This same model without reflection yielded $\chi_{\nu}^{2}=1.33 / 348$ dof and $k T=17.7 \pm 0.4 \mathrm{keV}$. If we allow the Fe abundance to vary, a multi-temperature model without reflection yielded $k T_{\max }=18.2 \pm 0.5 \mathrm{keV}$ and an $\mathrm{Fe}$ abundance of $0.87 \pm 0.05 \mathrm{Fe}_{\odot}$ with $\chi_{\nu}^{2}=1.06 / 347$ dof. Including

\footnotetext{
9 Throughout our spectral analysis, the derived elemental abundances refer to the solar abundances from Anders \& Grevesse (1989).
} 
Table 1

Spectral Fit Results

\begin{tabular}{|c|c|c|c|c|c|}
\hline Data & Model & $\chi_{\nu}^{2} /$ dof & $k T_{\max }[\mathrm{keV}]$ & $\mathrm{Fe} / \mathrm{Fe}_{\odot}$ & Amplitude \\
\hline NuSTAR & brems $\times$ ref & $1.27 / 212$ & $9.9 \pm 0.6$ & 1 & $\lesssim 0.29$ \\
\hline NuSTAR & brems $\times$ ref & $1.27 / 212$ & $9.9 \pm 0.6$ & 0.6 & $\lesssim 0.29$ \\
\hline NuSTAR & vmcflow $\times$ ref & $1.19 / 347$ & $13.4 \pm 0.8$ & 0.6 & $1.24 \pm 0.33$ \\
\hline NuSTAR & vmcflow & $1.33 / 348$ & $17.7 \pm 0.4$ & 0.6 & $\cdots$ \\
\hline NuSTAR & vmcflow & $1.06 / 347$ & $18.2 \pm 0.5$ & $0.87 \pm 0.05$ & $\cdots$ \\
\hline NuSTAR & vmcflow $\times$ ref & $1.06 / 346$ & $18.3_{-0.6}^{+0.4}$ & $0.87 \pm 0.04$ & $\lesssim 0.08$ \\
\hline$N u S T A R+$ Swift + Chandra & vmcflow $\times$ ref & $1.17 / 1900$ & $19.7 \pm 0.4$ & $0.88 \pm 0.02$ & $\lesssim 0.15$ \\
\hline NuSTAR + Swift + Chandra & vmcflow & $1.17 / 1989$ & $19.7 \pm 0.4$ & $0.88 \pm 0.02$ & $\cdots$ \\
\hline
\end{tabular}

reflection, the fit, with $\chi_{\nu}^{2}=1.06 / 346$ dof, yielded $k T_{\max }=$ $18.3_{-0.6}^{+0.4} \mathrm{keV}, \mathrm{Fe} / \mathrm{Fe}_{\odot}=0.87 \pm 0.04$ and reflection amplitude $\lesssim 0.08$.

Because the reflection amplitude depends on the $\mathrm{Fe}$ abundance, and the NUSTAR data with their low spectral resolution are well suited to fit the continuum but not the spectral lines, we also included the Chandra HETG and Swift data in the fit of our multi-temperature model. For the Chandra data, we only used the $3.0-8.0 \mathrm{keV}$ energy range; lower energies are dominated by soft emission lines that are not adequately described by isobaric, multi-temperature spectral models (Mukai et al. 2003; Luna et al. 2015). We let the NuSTAR data drive the fit of the reflection and cooling flow temperature, while the Chandra data drove the Fe abundance. The fit led to a $\chi_{\nu}^{2}=1.17 / 1900$ dof, $k T=19.7 \mathrm{keV}, \mathrm{Fe}$ abundance of $\mathrm{Fe} / \mathrm{Fe}_{\odot}=0.88$ and an unconstrained reflection amplitude of $\lesssim 0.15$. Once we consider the same model without reflection, we have $k T=19.7 \mathrm{keV}, \mathrm{Fe} / \mathrm{Fe}_{\odot}=0.88$ and $\chi_{\nu}^{2}=$ $1.17 / 1899$ dof (see Figure 1). No differences were found from the previous model, owing to the undetectable reflection. The strength of the Compton hump due to reflection will be small if we see the reflection surface edge-on, which will imply that the parameter $\cos (\mu)$ is closer to zero. We tried models with low $\cos (\mu)$ of 0.1 and found that the shock temperature and the negligible reflection amplitude are insensitive to the value of $\cos (\mu)$, reinforcing our contention that the reflection component is weak or absent.

\subsection{The Uncontaminated Hard X-Ray Flux}

The best-fit spectral model from NuSTAR + Swift + Chandra data yielded a $12-40 \mathrm{keV}$ flux $3.3 \times 10^{-11} \mathrm{erg} \mathrm{s}^{-1} \mathrm{~cm}^{-2}$ and when this model was applied to the $S u z a k u / H X D$ data, it yielded a $12-40 \mathrm{keV}$ flux $3.9 \times 10^{-11} \mathrm{erg} \mathrm{s}^{-1} \mathrm{~cm}^{-2}$. Yuasa et al. (2010) quoted a $12-40 \mathrm{keV}$ flux of $3.56 \times 10^{-11} \mathrm{erg} \mathrm{s}^{-1} \mathrm{~cm}^{-2}$ from their modeling of Suzaku data. The difference can be attributed to the high absorption column quoted by Yuasa et al. (2010; see their Table 2). We conclude that the real hard energy flux has been contaminated by $\approx 20 \%$. Our model also yielded a mass accretion rate $\dot{M}=1.89 \times 10^{-11} M_{\odot} \mathrm{yr}^{-1}$. Note that while modeling the Chandra spectrum, Luna et al. (2015) used $\dot{M}$ of $1.74 \times 10^{-11} M_{\odot} \mathrm{yr}^{-1}$, while Isakova et al. (2017) used $\dot{M}$ of $4.75 \times 10^{-11} M_{\odot} \mathrm{yr}^{-1}$ in their numerical simulations of the accretion flow.

\subsection{Timing Analysis: Power Spectrum and Pulsed Fraction}

In order to study the dependence of the WD spin-pulsed fraction with energy from the photon arrival times we calculated the $Z_{1}^{2}$ (Rayleigh) statistic (Buccheri et al. 1983) as a function of frequency in the range $0.00022 \mathrm{~Hz}<f<$ $0.0003 \mathrm{~Hz}$ (the WD spin period is 67.02696576 minutes or $0.00024865614 \mathrm{~Hz}$; Mauche et al. 2009) with a step $\Delta f=1.0 /(T \times q)$, where $T$ is the exposure time and $q$ is the oversampling factor, which we took to be equal to 1000 . As the Swift data do not cover a single spin period, we did not include them in this analysis. The value of $Z_{1}^{2}$ needed to detect a pulsation with a probability $P=2.699 \times 10^{-3}$ ( $3 \sigma$ detection) is $Z_{1}^{2}>2 \ln \left(\frac{T \Delta f}{P}\right)$. If the peak in the power spectrum is due to nearly sinusoidal modulations, the pulsed fraction is $p=p_{\text {obs }}\left(N_{S}+N_{B}\right) N_{S}^{-1}$, where $N_{S}$ and $N_{B}$ are the number of source and background counts and $p_{\text {obs }}$ is the observed pulsed fraction uncorrected by the background. However, as EX Hya is a very bright X-ray source, the background contribution is negligible and the pulsed fraction can be expressed as $p \simeq \sqrt{2 Z_{1}^{2}} N_{S}^{-1 / 2} \pm 2 N_{S}^{-1 / 2}$.

In Figure 2 we plot the pulsed fraction of those spin periods detected with $>3 \sigma$ significance in the energies of the strongest emission lines as observed in the Chandra/HETG spectra (and whose fluxes were measured in Luna et al. 2015) and the broad energy bands of 3-6, 6-9, and 9-12 keV in the NuSTAR data. Modulation at the spin period is detected up to energies of less than $\sim 12 \mathrm{keV}$ in the NUSTAR data. We found that there is a dependence of the pulsed fraction with energy. For energies of less than about $1 \mathrm{keV}$, the pulsed fraction seems to be constant with energy. On the other hand, for energies greater than $1 \mathrm{keV}$, the pulsed fraction decays with energy. The low absorption cannot be responsible for the modulation at these energies The origin of this effect remains a mystery. The non-detection of pulsation at energies greater than $\sim 12 \mathrm{keV}$ indicates that the height of the shock is at least greater than $1 R_{\mathrm{WD}}$ but not big enough to get the lower pole occulted by the inner region of the accretion disk.

We can qualitatively understand the energy dependence of spin modulation amplitude as a consequence of the height dependence of both the physical condition and the visibility. In the post-shock region, the temperature is at its highest near the shock and declines toward the white dwarf surface. The density, on the other hand, is at its lowest near the shock and increases near the surface. Continuum photons of energy $E$ originate from regions where $k T \gtrsim E$; line photons originate from a limited range of temperatures (e.g., Ne $\mathrm{X}$ lines require temperatures of the order of $0.54 \mathrm{keV}$; neither regions that are too cool or too hot contribute significantly). Thus, photon energy plotted along the $x$-axis of Figure 2 is a proxy for the origin of these photons within the post-shock region. If the shock is tall, of the order of $h_{\mathrm{sh}} \sim 1 R_{\mathrm{WD}}$, then the highest temperature continuum will escape self-occultation almost 


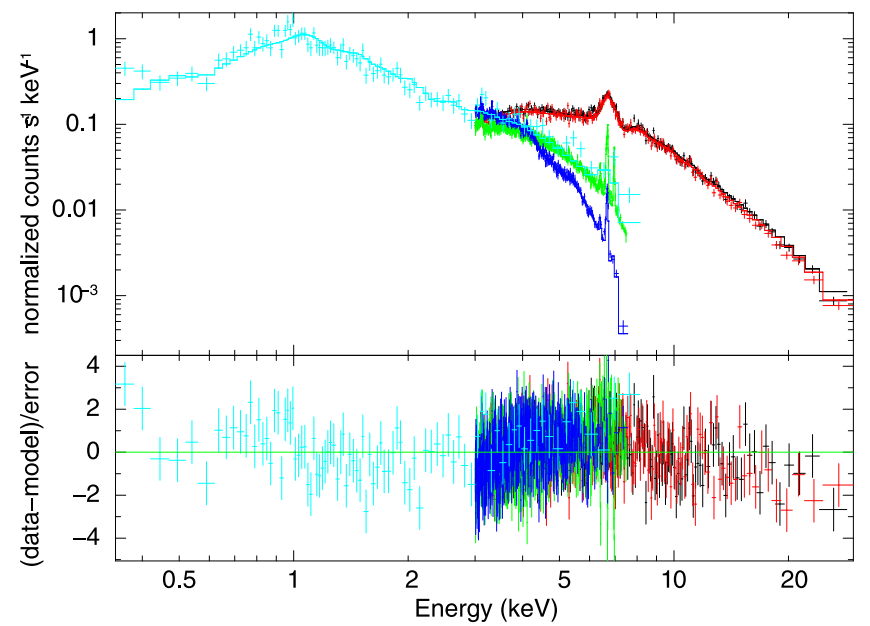

Figure 1. NUSTAR (black and red) + Swift (light blue) + Chandra (MEG, blue and HEG, green) EX Hya spectra modeled with a multi-temperature, variableabundances, isobaric cooling flow model, plus a fluorescent $\mathrm{Fe} \mathrm{K} \alpha$ emission line (constant $\times$ TBabs $\times$ reflect $\times($ vmkcflow+Gauss $))$. The lower panel shows the fit residuals in units of $\chi^{2}$.

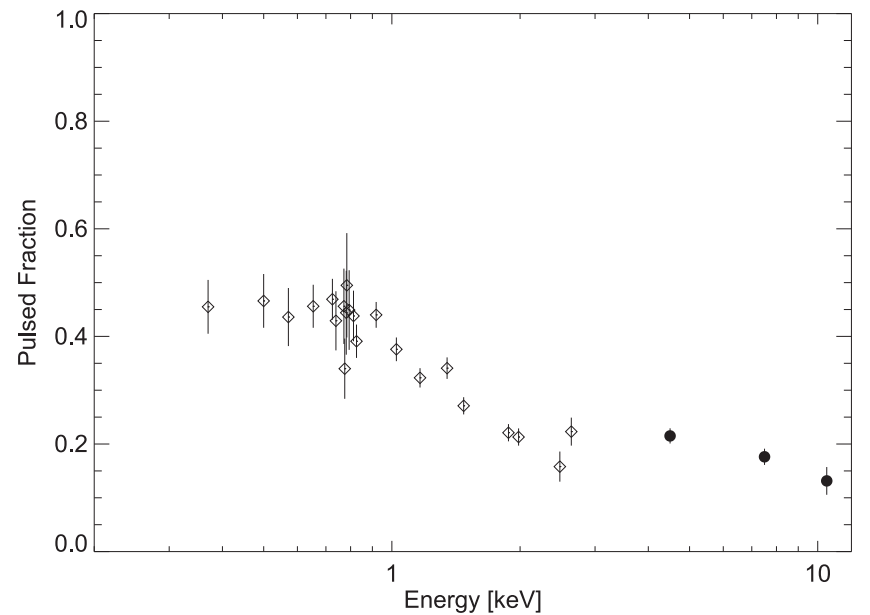

Figure 2. Pulsed fraction vs. energy derived from the NuSTAR observation in the energy intervals of 3-6, 6-9, and 9-12 keV (filled circles). The open diamonds show the pulsed fraction derived from the emission lines in the Chandra HETG spectrum. The lines are those listed in Table 1 in Luna et al. (2015).

completely. The lowest energy lines are emitted only near (but still above) the white dwarf surface. When we view the poles at a right angle, both poles are visible; half a spin cycle later, most of the lower pole is behind the body of the white dwarf, with a small residual that depends on the geometrical extent of the accretion footpoint. In Figure 3, we show the result of a proofof-concept simulation, in which light curves for uniform emission regions with a limited range of $h_{\mathrm{sh}}$ have been simulated. Following the two-stage process explained in Mukai (1999), the arc-shaped accretion footpoints were calculated assuming a rigid magnetic dipole with a magnetic colatitude of $5^{\circ}$, accreting uniformly from a transition region at the inner disk edge at $9-10 R_{\mathrm{WD}}$ (each pole accreting from an $180^{\circ}$ azimuth). A quantitative model including the location and shape of the threading region, and the resulting shock structure, is beyond the scope of this paper.

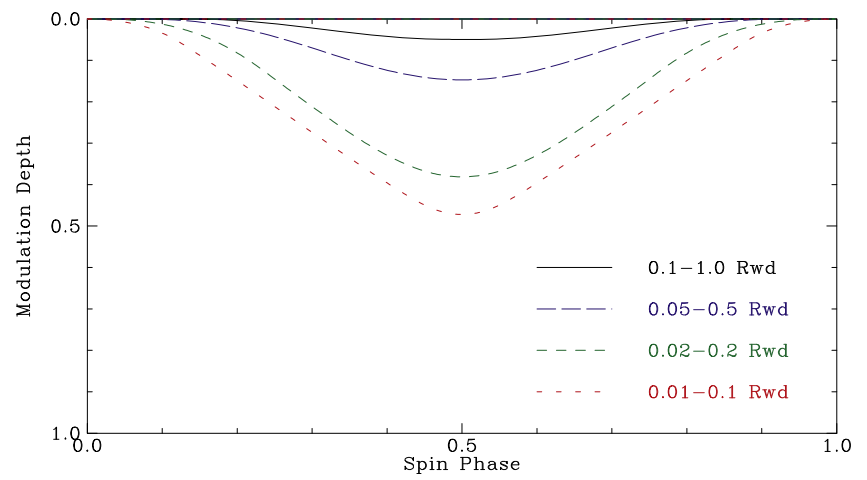

Figure 3. Modulation depth for different heights in the post-shock region.

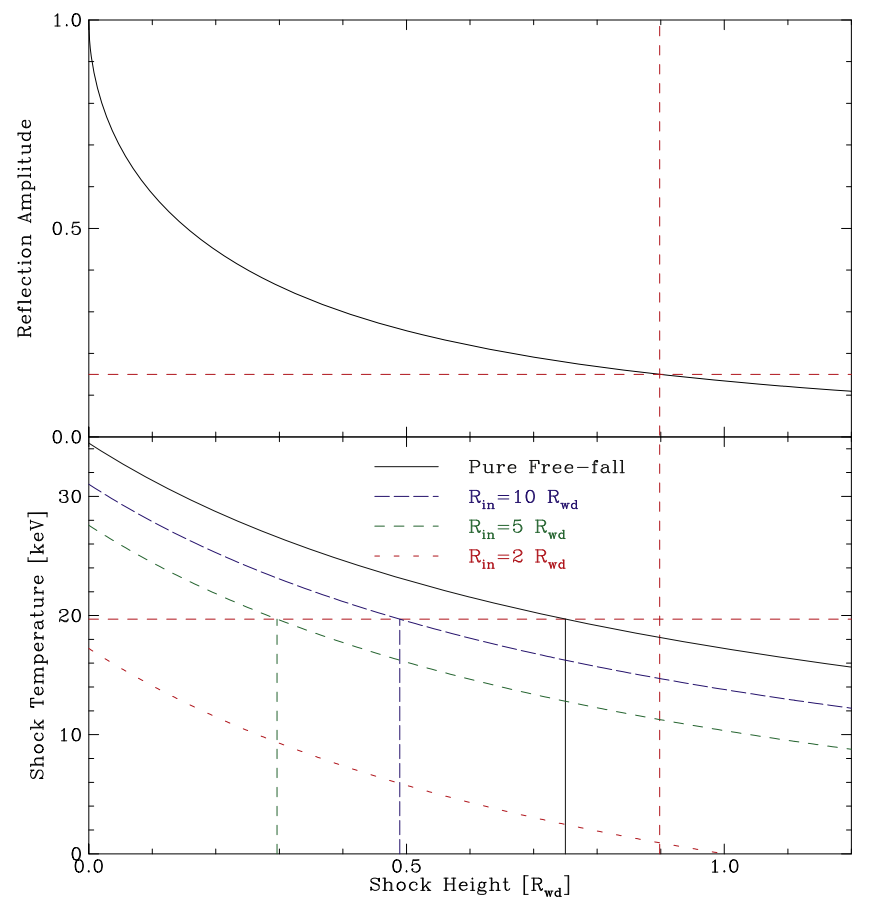

Figure 4. Top: the expected reflection amplitude as a function of $h_{\mathrm{sh}}$. Bottom: $k T_{\mathrm{sh}}$ as a function of $h_{\mathrm{sh}}$, assuming four different values of $R_{\mathrm{in}}$, including pure freefall, and a WD mass of $0.78 M_{\odot}$. The horizontal line at $19.7 \mathrm{keV}$ marks the $k T_{\text {sh }}$ that we measured (see Section 3).

\section{Discussion and Conclusions}

The non-detection of a reflection component in the hard $\mathrm{X}$-ray spectrum taken with $N U S T A R$, the small $\mathrm{Fe} \mathrm{K} \alpha$ equivalent width of $\sim 25 \mathrm{eV}$, and the absence of spin modulation for energies greater than $\sim 12 \mathrm{keV}$ implies that $h_{\mathrm{sh}}$ must be an appreciable fraction of the WD radius. This can be used to distinguish between the two possible origins (large $h_{\text {sh }}$ and small $R_{\text {in }}$ ) of low $k T_{\text {sh }}$ in EX Hya.

In Figure 4, the top panel shows the reflection amplitude as a function of $h_{\mathrm{sh}}$ for a point-like emitting region. The upper limit on the reflection amplitude of $\lesssim 0.15$ (see Table 1) implies $h_{\text {sh }} \lesssim 0.9 \times R_{\mathrm{WD}}$. Also, the EW of FeK $\alpha$ implies a reflection amplitude of about 0.15 in the model presented by George \& Fabian (1991). The bottom panel in Figure 4 shows a set of four curves of $k T_{\mathrm{sh}}$ as a function of $h_{\mathrm{sh}}$, for four different assumed values of $R_{\text {in }}$, for a $0.78 M_{\odot}$ white dwarf, with a horizontal line at $19.7 \mathrm{keV}$, the $k T_{\mathrm{sh}}$ that we measured. Accounting for the error bar in the $k T_{\mathrm{sh}}$, the reflection amplitude upper limit, and our simplified reflection model, 
the data presented here are compatible with a large $R_{\text {in }}$. Moreover, given the lack of strong reflection signature, a solution of $R_{\mathrm{in}}=2 \times R_{\mathrm{WD}}, h_{\text {shock }}=0$ is clearly untenable for EX Hya.

The inferred large $R_{\text {in }}$ is still much smaller than the co-rotation radius for a WD rotating with a 67-minute period. In the diamagnetic blob scenario proposed by King \& Wynn (1999) and Norton et al. (2008), which implies a perturbed accretion flow that deviates from purely Keplerian velocity structure, it is possible that the WD is in spin equilibrium. Note that the magnitude of the spin-up in EX Hya does not stand out among all IPs, most of which are presumably in spin equilibrium. Moreover, in this scenario, the very premises of the model of stochastic variability used by Revnivtsev et al. (2011) and Semena et al. (2014), as well as other determinations of $R_{\text {in }}$ based on strictly Keplerian flows, are suspect. Alternatively, EX Hya may possess a purely Keplerian accretion disk with a large $R_{\mathrm{in}}$ : in this case, the WD is far out of equilibrium, and we have no concrete explanation for the disagreement with the break frequency method. One remaining problem that still needs to be explained is the detailed soft X-ray emission line spectroscopy in the Chandra HETG data.

This research has made use of data obtained with the NUSTAR mission, a project led by the California Institute of Technology (Caltech), managed by the Jet Propulsion Laboratory (JPL) and funded by NASA. M. Orio acknowledges support from NASA grant NNX17AB76G. We acknowledge the Swift team for planning this observation. G.J.M.L. is a member of the CIC-CONICET (Argentina) and acknowledges support from grants PIP-Conicet/2011 \#D4598, ANPCYTPICT 0478/14.

Facilities: NuSTAR, Swift, and Chandra.

\section{ORCID iDs}

G. J. M. Luna (iD https://orcid.org/0000-0002-2647-4373

K. Mukai (D) https://orcid.org/0000-0002-8286-8094
M. Orio (10) https://orcid.org/0000-0003-1563-9803

P. Zemko (iD https://orcid.org/0000-0001-8408-0420

\section{References}

Aizu, K. 1973, PThPh, 49, 1184

Allan, A., Hellier, C., \& Beardmore, A. P. 1998, MNRAS, 295, 167

Anders, E., \& Grevesse, N. 1989, GeCoA, 53, 197

Belle, K. E., Howell, S. B., Sion, E. M., Long, K. S., \& Szkody, P. 2003, ApJ, 587, 373

Buccheri, R., Bennett, K., Bignami, G. F., et al. 1983, A\&A, 128, 245

Echevarria, J., Ramirez-Torres, A., Michel, R., \& Hernandez Santisteban, J. V. 2016, MNRAS, 461, 1576

Gastaldello, F., Ettori, S., Molendi, S., et al. 2003, A\&A, 411, 21

George, I. M., \& Fabian, A. C. 1991, MNRAS, 249, 352

Hayashi, T., Kitaguchi, T., \& Ishida, M. 2018, MNRAS, 474, 1810

Hellier, C., Mason, K. O., Rosen, S. R., \& Cordova, F. A. 1987, MNRAS, 228, 463

Isakova, P. B., Zhilkin, A. G., Bisikalo, D. V., Semena, A. N., \& Revnivtsev, M. G. 2017, ARep, 61, 560

King, A. R., \& Lasota, J. P. 1991, ApJ, 378, 674

King, A. R., \& Wynn, G. A. 1999, MNRAS, 310, 203

Luna, G. J. M., Raymond, J. C., Brickhouse, N. S., et al. 2010, ApJ, 711, 1333

Luna, G. J. M., Raymond, J. C., Brickhouse, N. S., Mauche, C. W., \& Suleimanov, V. 2015, A\&A, 578, 15

Mauche, C. W., Brickhouse, N. S., Hoogerwerf, R., et al. 2009, IBVS, 5876

Mukai, K. 1999, in ASP Conf. Ser. 157, Annapolis Workshop on Magnetic Cataclysmic Variables, ed. C. Hellier \& K. Mukai (San Francisco, CA: ASP), 33

Mukai, K., Kinkhabwala, A., Paterson, J. R., Kahn, S. M., \& Paerels, F. 2003, ApJL, 586, L77

Mukai, K., Rana, V., Bernardini, F., \& de Martino, D. 2015, ApJL, 807, L30

Norton, A. J., Butters, O. W., Parker, T. L., \& Wynn, G. A. 2008, ApJ, 672,524

Revnivtsev, M., Potter, S., Kniazev, A., et al. 2011, MNRAS, 411, 1317

Semena, A. N., Revnivtsev, M. G., Buckley, D. A. H., et al. 2014, MNRAS, 442,1123

Siegel, N., Reinsch, K., Beuermann, K., Wolff, E., \& van der Woerd, H. 1989, A\&A, 225, 97

Suleimanov, V., Doroshenko, V., Ducci, L., Zhukov, G. V., \& Werner, K. 2016, A\&A, 591, 35

Suleimanov, V., Revnivtsev, M., \& Ritter, H. 2005, A\&A, 435, 191

Warner, B. 1972, MNRAS, 158, 425

Yuasa, T., Nakazawa, K., Makishima, K., et al. 2010, A\&A, 520, 25 